\title{
Further Structural Intelligence for Sensors Cluster Technology in Manufacturing
}

\section{Samir Mekid}

The University of Manchester, School of Mechanical, Aerospace and Civil Engineering. Sackville Street Building C51k, Po Box 88, Manchester, UK. E-mail: s.mekid@manchester.ac.uk Received: 18 April 2006 / Accepted: 13 June 2006 / Published: 15 June 2006

\begin{abstract}
With the ever increasing complex sensing and actuating tasks in manufacturing plants, intelligent sensors cluster in hybrid networks becomes a rapidly expanding area. They play a dominant role in many fields from macro and micro scale. Global object control and the ability to self organize into fault-tolerant and scalable systems are expected for high level applications. In this paper, new structural concepts of intelligent sensors and networks with new intelligent agents are presented. Embedding new functionalities to dynamically manage cooperative agents for autonomous machines are interesting key enabling technologies most required in manufacturing for zero defects production.
\end{abstract}

Keywords: intelligent sensors, agent, cluster network, hybrid network, manufacturing.

\section{Introduction}

Intelligent or smart sensors have been known for more than two decades. These sensors are more sophisticated than traditional sensors as they gather, analyse and transmit data. A state-of-the-art of intelligent sensors covering the last two decades are found in [1,2]. According to IEEE 1451.2 specifications, a smart sensor is a version of smart transducer that provides functions beyond those necessary for generating a correct representation of a sensed or controlled quantity. This functionality typically simplifies the integration of the transducer into applications in a networked environment.

The basic principle of an intelligent (i.e. smart) sensor is that its complexities must be concealed internally and must be transparent to the host system. Smart sensors are designed to present a simple face to the host structure via a digital interface, such that the complexity is borne by the sensor and not 
by the central signal processing system [3]. So far, an exact definition is still indefinite. However, generic concept of an intelligent sensor can be described according to Schodel [4]: 'It is common to call a sensor intelligent, if just a microprocessor device is assembled at the location of the sensor transducer, to implement filtering and other simple pre-processing tasks at the location of the sensor'. In addition to the previous concept, Brignell [5] describes intelligent sensor that 'modifies its internal behaviour to optimise its ability to collect data from the physical world and communicate them in a responsive manner to a host system'. This concept is similar to the concept given by Chita [6]. The ultimate intention of developing intelligent sensors is to imitate human abilities such as multiple functions for sensing objects simultaneously, learning with adapting capabilities and decision making [7]. The development of intelligent sensors rests on advances in hardware (i.e. measurement technology) and advances in software (i.e. processing technology). Advancing in microelectronic, microcomputer and manufacturing technologies enables an integration of sensing elements and signal processing elements embedded into a single chip found for example in Micro-Electro-Mechanical System (MEMS). For multiple measurements, Nagel [8] introduced the concept of 'cluster'; the integral of several different sensing elements uses common computing and communicating capabilities and shares a power supply to imitate the human abilities. Continuous improvement in MEMS technology can ease this implementation. The use of such sensors is very promising in manufacturing as there is a clear trend towards modularity of future holistic intelligent Computer Numerically Controlled Machines (CNC), PLCs and Robots based on distributed control design which allows flexible control configuration and adaptation of systems. Such systems are governed by intelligent control systems consisting of a hierarchical structure of production control, machine control and drive control layers that have to implement open interfaces, learning capabilities, self-tuning mechanisms and sophisticated model-based prediction instruments in order to allow automated error-free machining for example. The future multi process autonomous machine is expected to be equipped with new concepts of multi agent sensors cluster to enable a full control of diverse variables such as cutting parameters, in-process dimensional measurement, geometric and form defects (Figure 1). After a static, dynamic and thermal analysis, a sensor mapping strategy on the machine is of paramount importance to ensure continuous and accurate feedback. As an example, various wireless sensors are placed at high amplitudes locations of major modes of vibrations, while thermal sensors are located in critical locations subject to larger expansions. With such a sensor mapping, it is planned to implement compensation of errors and forces while machining to secure zero defect workpiece in the autonomous mode.

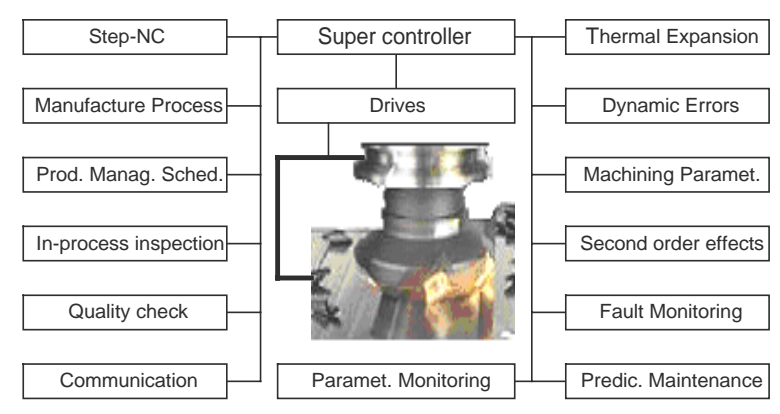

Figure 1. Error mapping in machine tools. 
The use of reconfigurable sensors for maintenance requires data to participate in feature recognition in order to help in the identification of possible failures types and immediately decide on the actions to be taken (self diagnostic and self service strategy, self healing requiring autonomous supervision).

In large complex plants such as chemical applications requiring safety and reliability, the processes are based on highly automated control systems involving a large number of sensors. These should be reconfigurable and capable of performing data interpretation and fusion from multiple sensors with the validation of local and remotely collected data. This will help in the reduction of false alarms and downtime as well as avoid jeopardizing personnel and environmental safety. Moreover, the data generated by manufacturing plants requires suitable techniques to improve their accuracy and to extract useful information about the operational status of the process.

To enable such remarkable features in sensors technology, a number of core characteristics are presented and discussed next.

\section{2- Structure and functions of an intelligent sensor}

\subsection{Intelligent sensor architecture}

As illustrated in Fig. 2, intelligent sensor architecture comprises the following;

(a) A sensing element that links the external world to a sensor system by generating electrical signal (e.g. voltage, current) with response to physical properties of the environment such as temperature, pressure, light intensity, sound, vibration, etc.

(b) An interface element for signal conditioning and data conversion. The signal obtained from the sensing element is modified, enhanced and converted to a discrete time digital data stream before passing through a processing element.

(c) A processing element that includes a microcontroller with an associated memory and software; this is the main component of the architecture where the incoming signal is processed.

(d) A communication element, which provides a two-way communication between the processing element and users. The communication are wireless, optical fibres, serial buses, and interfacing to successful communication with the outside world.

(e) A power source.

The sensing part within an intelligent sensor may differ from conventional transducer material. As hysteretic and nonlinear behaviour materials were previously discarded because of non-reliability or instability in sensing applications, they could be used in a sensor that contains its own dedicated microprocessor; the need to burden a central processor with a complex model or filtering algorithm is thereby avoided. Applications can be envisioned exploiting the inherent memory or hysteresis of nonlinear materials to reduce the signal processing workload for example, "record" peak temperature [3]. The signal processing concept present in a smart sensor will relieve the constraint of the paradigm that sensor elements must be linear and noise-free; however, the cost of the added electronics must be considered in the sensor system design analysis. Two architectures are shown in Fig. 2 (a) and (b). The former integrates all elements into a single structure whereas the latter separates sensing and interface elements from processing and communication parts. 


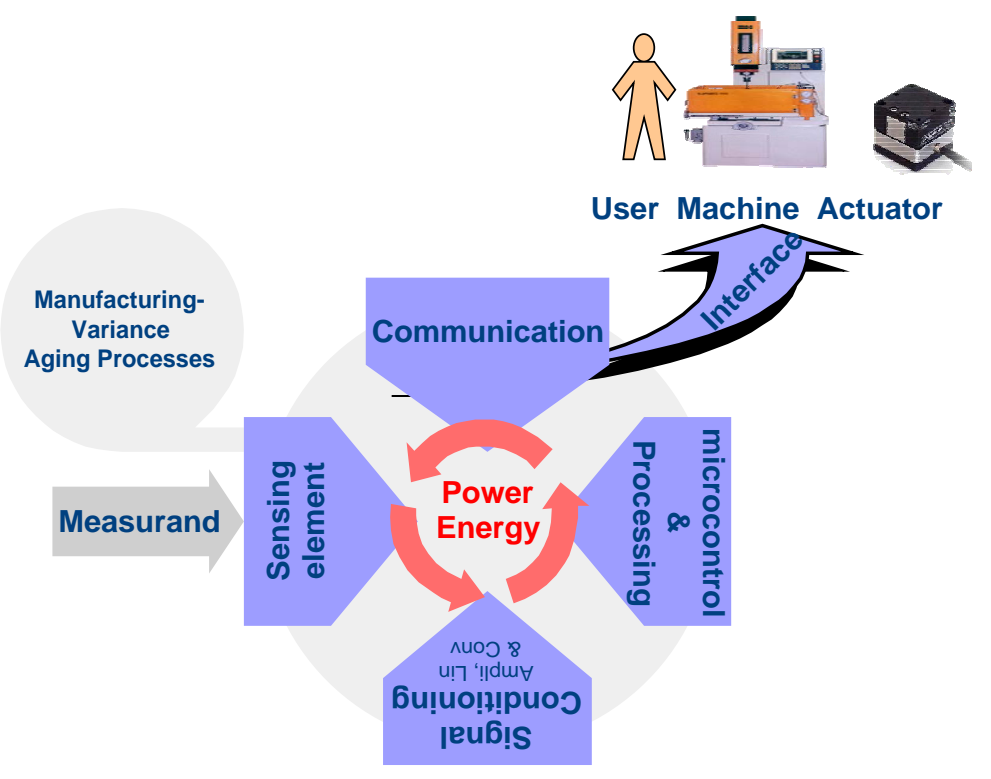

(a)

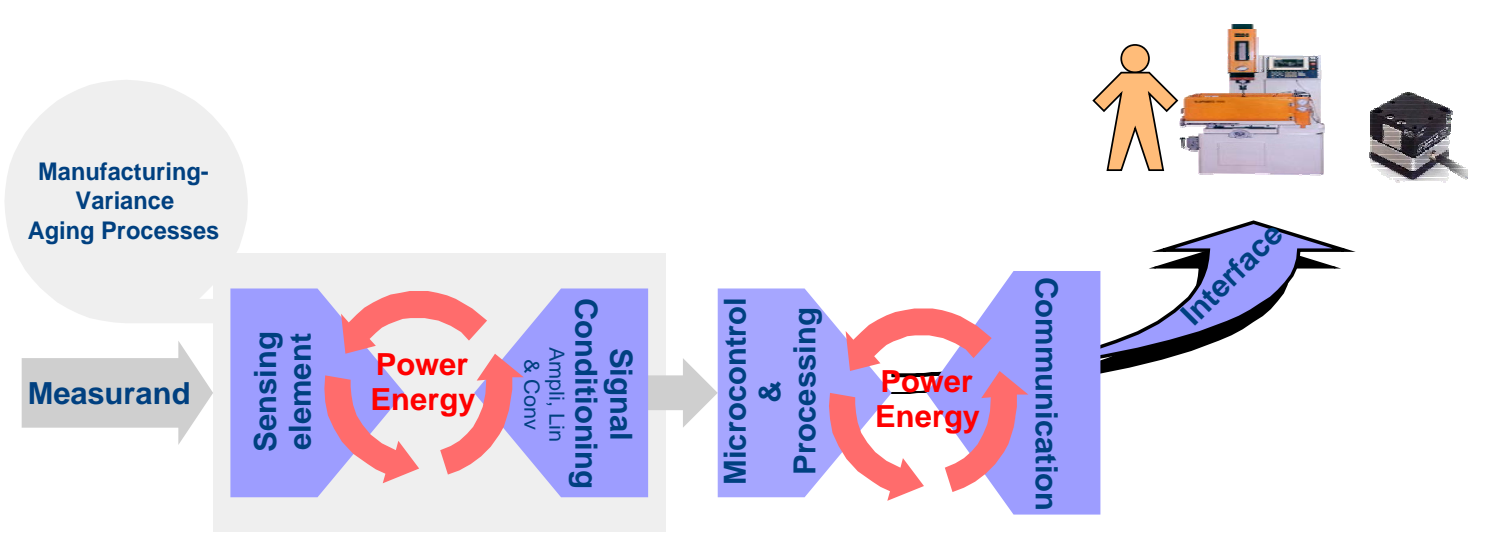

(b)

Figure 2. Intelligent sensor architecture;

(a) Integrated intelligent sensor, (b) Semi-integrated intelligent sensor.

The semi-integrated intelligent sensor architecture has been proposed according to existing sensors claiming intelligence. The development of an intelligent sensor system is limited by the components limitation such as battery lifetime for wireless sensors and the limited memory with low processor speed that have an effect on the processing unit performance. Extensive research has been carried out to develop fully integrated smart sensors with smaller size sensing element and to improve precision and accuracy of measurements. Improving power consumption requirement and the design of selfpowered sensors which energy is converted from mechanical vibration [9, 10,11, 12] or from light source [13] are also of interest. Moreover, the development in software programs to include more expert systems is vital to the improvement of intelligent sensors. To extend the use of those sensors in complex systems with difficult data transmission, wireless communication [14] was introduced with 
the support of bluetooth, WIFI, optical fibre technologies and fieldbus [15]. Potential advantages of the intelligent sensor concept include:

- Reduced down time.

- Fault tolerant systems.

- Adaptability for self-calibration and compensation.

- Higher reliability.

- Master/Slave sensors mapping capability.

- Lower weight.

- Lower cost.

- Lower maintenance.

\subsection{Intelligent sensor functionalities}

An intelligent sensor is expected to demonstrate multi-channel sensing, store sensing signals to be of later use, be able to make decision according to stored information and adapt itself to changing conditions. Various functionalities have been suggested for intelligent sensors. Robert [16] proposed that the intelligent sensors should encompass configuration, communication, measurement, computing and validation functionalities. Similarly, Meijer [17] includes three functionalities; compensation, computing and communication. While Tian [18] suggested that what is called an intelligent sensor should have the functions of compensation, validation, data-fusion and communication. Despite the difference in categorisation, these functionalities are overlapped and complimentary to each other. New functions such as self-calibration could be also addressed. Therefore, the generic functionalities of intelligent sensors are summed up as follows:

\subsubsection{Compensation functionality}

The compensation is a method to improve measurements for better accuracy by considering the errors in the system. The task is easier if systematic errors are known. The compensation could be divided into three categories:

(a) Non-linear compensation that linearises the relationship between input and output.

(b) Cross-sensitivity compensation such as temperature control compensation.

(c) Time based or long term drift compensation due to degradation of the sensor elements [18].

Compensation functionality confirms the sensor performance and ensures that sensors are working correctly. Self-diagnostic, self calibration [19-23] and adaptation are applied into intelligent sensors to detect and eliminate the influence of systematic errors and to check the sensor performance. The numerical methods applied are sometimes onerous.

\subsubsection{Processing functionality}

Processing is the ability to provide the most relevant information in an efficient representation to the communication interface. This processing task involves signal conditioning, signal conversion, logic functions, data reduction that is used to enhance the received signal, and, decision making in conjunction with other signals types from other sensors (i.e. single sensor or sensors cluster). The 
measured quantity is determined despite the effects of manufacturing variance, environmental parameters and ageing processes. Development in software technology enhances the computing capability with the use of artificial neural network, neuro-fuzzy or neuro-genetic to try imitating the ability of human brain.

\subsubsection{Communication functionality}

The purpose of the communication functionality is to exchange information between the sensor and the user and to allow users to reprogram for other sets of measurement. In this case, a two-way communication is needed. The communication can pass through either wires, optical fibres, wireless or fieldbus options. These developing communication technologies make communication systems less complex with better flexibility at low cost. An example of communication functionality is described in [16]. Further details are given in §2.3.

\subsubsection{Validation functionality}

Data validation increases the overall reliability of sensors system. Faulty data can cause unexpected behaviour or system failure; therefore, an intelligent sensor must be able to evaluate the validity of the collected data to avoid any disastrous effects of the propagation of erroneous data and to warn users about it. The impact of such errors may be reduced with a dense sensors network, or, with a knowledge based system where the smart sensor incorporates expert systems for example. Self-validation becomes more important when various data from multi-sensors or multi-measurements are sent to the system [18, 24]. The objectives of this validation are to enhance safety, to provide better product quality, to reduce unscheduled shutdowns and to improve plant efficiency and availability.

\subsubsection{Integration functionality}

This concerns the integration of the sensing element with data processing and communication on a single ship to eliminate wires connection between components, to reduce the overall size of the sensors, to optimally use the power energy and to reduce costs. Therefore, the downscale of such sensors will ease their use in clusters and hence increases the overall efficiency and reliability of measurements. MEMS is an enabling technology that allows the development of smart products. It augments the computational ability of microelectronics with the perception and control capabilities of micro-sensors and micro-actuators and expands the space of possible designs and applications. More implementations are expected with nanotechnology and biotechnology.

\subsubsection{Data fusion functionality}

The function of data fusion is to ensure that only the most relevant information is transmitted between sensors. Information from multiple sensors or multi-measurements can be combined, associated and correlated using data fusion techniques. The latter can be categorised into three architectures; centralised fusion, decentralised fusion and hybrid fusion. The centralized fusion architecture combines multi-sensor data while it is still in its raw form. Unlike the centralized fusion 
architecture, the decentralized fusion architecture allows each sensor to perform feature extraction before the fusion process. The combination of the centralised and decentralised approaches provides a hybrid architecture that fuses raw data, feature data and decision data [25].

\section{3- Interface with the outside world}

Interfacing is one of the most important issues found in industry related to intelligent process instrumentation e.g. sensors and actuators. Hence not only field-bus based smart sensors design and application are rapidly becoming the preferred choice for building the next generation of distributed measurement and control systems, but also Internet technologies such as TCP/IP with Ethernet, Bluetooth and WIFI. The framework which can support the current trend comes from IEEE 1451 standards specifying smart transducer interface architecture that enables to unify not only interconnecting smart sensors with various field-buses but also direct coupling to the Ethernet-based Intranet. IEEE 1451 consists of the family of standards for a networked smart transducer interface that include namely a smart transducer information model; 1451.1, targeting software-based, network independent, transducer application environments and a standard digital interface and communication protocol. 1451.2 [26] is used for accessing the transducer via the microprocessor modelled by the 1451.1. The next two standards, 1451.3 and 1451.4 extend the possible single-attached configurations to the embedded distributed multi-drop systems and to mixed-mode communication protocols for analogue transducers.

An important phenomenon in the implementation of such interface standards is the enormous growth of complexity of control and data acquisition systems. IEEE-1451.2 was the first standard to be finalized [27]. Its mission was to separate the network issues from the transducer issues. This was accomplished with four concepts: the Smart Transducer Interface Module (STIM), the Network Capable Applications Processor (NCAP), the Transducer Independent Interface (TII) and the most important element of this strategy the Transducer Electronic Data Sheet (TEDS). The STIM handled the sensor and actuator low-level interface information and formatted data communication messages between the NCAP and STIM in a standardized digital manner. The NCAP handled the network interface and also managed the TII dedicated interface port to the STIM. It can be argued that the TEDS was the crown jewel of this strategy in that it provided for self- identification of the connected sensor or actuator in a very general manner. Depending on how many of the TEDS fields are implemented, TEDS information can provide great details about how to read data from the transducer and help identify which transducer is being viewed [28].

\section{Systems characteristics and related agents}

\section{3-1 Systems characteristics}

Systems have different characteristics and requirements such as those within autonomous machines. The most suitable categories as defined in cognitive systems [29, 30] are defined hereafter:

a) Ill-structured: usually designed systems do not have all the necessary structural information, they are ill-structured. Agents have a distinct notion about themselves and know that their environment can change, hence suitability for a dynamic environment. 
b) Modular: an agent applies very well to modular systems as it has dedicated tasks.

c) Decentralised: an agent can easily be applied in decentralised system to ease self-decision making without interference with other processes.

d) Changeable: an agent is very good for changeable systems. Change could be handled by both modular and decentralised systems. Decentralisation minimises the impact one module has on another when it changes.

e) Complex: with the increased complexity of systems, the agent is very well suited to handle such difficult task to model the behaviour of one or several components within its responsibility.

f) Real-Time: real time control is required in various applications today where an agent is very suitable to achieve it.

To identify the emerging intelligence between agents, Nwana [31] has emphasised autonomy, learning, and co-operation as characteristics that are very important in an intelligent agent but he did not mention the word 'intelligence' itself. Figure 3 shows the emerged agents from the interaction between the characteristics as defined by Nwana.

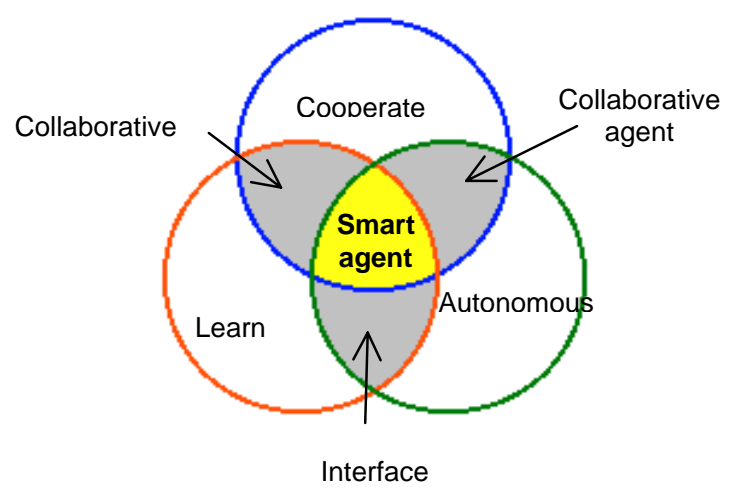

Figure 3. An agent topology according to Nwana.

\subsection{Key agents}

A sensor agent is able to control and optimise the use of a sensor within the cluster or the distributed sensors system. Among the tasks, it could decide which sensor has the task to start measuring according to its location and appropriate time. It could be replaced in case of failure and the task immediately transferred to another equivalent sensor. The designed agent should have the ability to learn rapidly from the existing situation to decide which sensor should go for the current query. The agent could identify and memorise the more reliable sensors from low reliable ones and could learn as it progresses. With its local control, this agent can determine the efficiency of the overall measurement, learn, cooperate and communicate with other agents.

An object agent is the other new concept to be dedicated to the object being monitored. It conserves all the information about it. The user could request at any time the current object information. If an object is moving in a fluid, a series of sensors could track it as it passes in their respective action area and the information is compiled by the sensor agent for the abject agent. Laser trackers for moving objects are very good examples. 


\subsection{Intelligent agents}

The capabilities of the sensors are relatively limited by the degree of intelligence provided by the associated agents. Hence, it is important to identify the required agents as well as their structure to suit the above requirements. But according to [32] the definition of such agents is still not clear among the interested community. "Some have tried to offer the general definition of agents as someone or something that acts on one's behalf, but that seems to cover all of computers and software. Other than such generalities, there has been no consensus on the essential nature of agents." [33]. A large list of definitions has already been proposed in the literature to include the following without privileging any of the definitions:

a) The Brustoloni Agent [34, 35], "Autonomous agents are systems capable of autonomous, purposeful action in the real world."

b) The SodaBot Agent [36], "Software agents are programs that engage in dialogs [and] negotiate and coordinate transfer of information."

c) The Maes Agent [37], "Autonomous agents are computational systems that inhabit some complex dynamic environment, sense and act autonomously in this environment, and by doing so realize a set of goals or tasks for which they are designed." Maes also mentions other agent characteristic such as fast, reactive, adaptive, robust, autonomous and "lifelike".

d) The Nwana agent [38], to identify the emerging intelligence between agents, Nwana has emphasised autonomy, learning, and co-operation as characteristics that are very important in an intelligent agent but he did not mention the word 'intelligence' itself. In figure 3 shows the emerged agents from the interaction between the characteristics as defined by Nwana.

e) The Foner agent [39], according to Foner an agent must have certain characteristics that must be fulfilled in some way or another for an agent to be called an intelligent agent. The characteristics mentioned are autonomy, personalization, risk and trust, domain, graceful degradation, co-operation, anthropomorphism and expectations.

f) The Petrie agent [33], a general description of an agent as someone or something that acts on ones behalf, is according to Petrie not a sufficient definition, since this description can be applied to all computers and software.

g) The Jennings and Wooldridge agent [30], according to Jennings and Wooldridge an agent must be autonomous, an agent is autonomous if it is capable of acting without any direct guidelines from either humans or other agents. This means that the agent itself has control over its own actions and behaviour, i.e. the agent encapsulates its behaviour and internal state. If an agent is compared to an ordinary object that also has an internal state we can see an important difference; that there is at least one method in an object that can be invoked by another component. This implicates that an object is not autonomous.

h) The Hayes-Roth agent [40], finally the following definition of an intelligent agent is presented by Hayes-Roth: "Intelligent agents continuously perform three functions: perception of dynamic conditions in the environment; action to affect conditions in the environment; and reasoning to interpret perceptions, solve problems, draw inferences, and determine actions." 


\subsection{Single-agent and Multi or hybrid agent systems}

In the application cited earlier where different types of measurements are required for different purposes, it is clear that agents have to either work alone or cooperate to achieve a task. The question could be whether a single agent could perform all the tasks or a concept of multi-agent has to be introduced. Communication between agents is important to transfer information with a capability of prioritization. With multi-agent systems, parallel computation could be used to ease the programming and to speed up the system robustness and scalability [41].

Multi agent systems would not probably be of systematic use if the system is not suitable. In some cases, it may introduce delays or will be an expensive solution. Further multi-agents are not suitable when used only to decentralise a system normally modelled as a centralised one. It is not advised to provide multi-agent solutions to non-suitable problems, it is foremost important to focus on the problems the multi-agents are meant to solve and not on the possible benefits [42].

Today the focus of agent research is more on multi-agent systems than on single agent systems, since agents that co-operate and/or communicate can solve much more complex tasks than just what one single agent is capable of [43]. Verification and validation of multi agents has already been addressed in [44]. Learning and autonomous capabilities should be part of the multi-agents strategy. Nahm and Ishikawa [45] have proposed a hybrid network of agents as shown in Fig.4, they employ a lightweight middle agent, called "interface agent" for continuous or discrete interactions between local agents (type 1), between a collection of local agents and a collection of remote agents (type 2), and between a local agent and a remote agent (type 3). Therefore, agent interactions are made only via the interface agent.

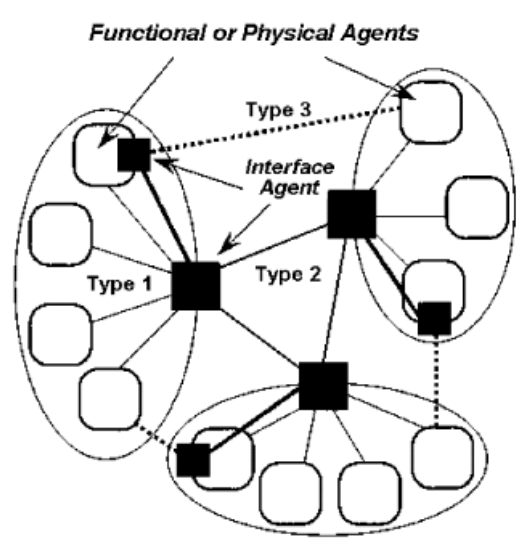

Figure 4. Hybrid network application.

In large sensors cluster with sub-system, this could be extended as we propose in figure 5 where (type 2') could be cooperative or central interaction that may be linked to another sub agents group with (type 4) between local agents of sub-groups and (type 5) between local agent and remote agent from other sub-groups. 


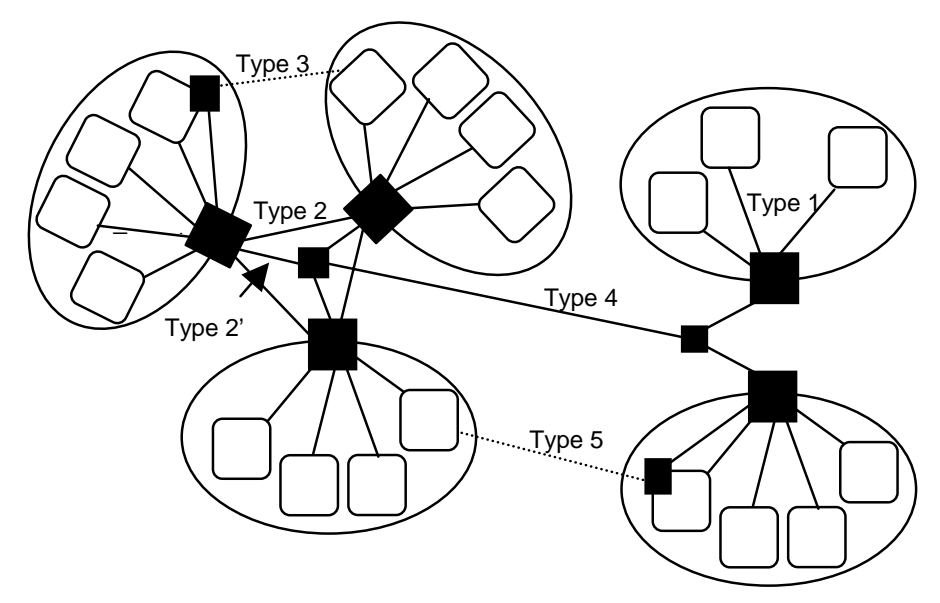

Figure 5: Proposed network application.

Passive sensors, as defined to simply measure and report on detected signals in their local environment, could also be used in conjunction with an intelligent cluster. These sensors are considered as slave sensors and their location in a system is very critical as the measurand has to be well defined and located. Layered hierarchical network structure could be a reasonable solution. Closer sensors will form a cluster that may report to a cluster master. This will also solve the scalability if the sensor network becomes very large.

\section{Applications of intelligent sensor and agents in manufacturing}

Application of intelligent sensors in manufacturing has been expanding over the past decade. A limited number of relevant applications of intelligent sensors in different manufacturing aspects are discussed. Tian [18] presented a fieldbus-based intelligent displacement frequency output sensor. It consists of a displacement frequency output sensor, a local operating network node and neural networks embedded in the neuron chip. The configuration diagram is shown in Fig. 6. The fieldbusbased intelligent sensor has functionalities of self-calibration, self-validation, self-compensation, computing and communication. Self-calibration and self-compensation are used to detect and eliminate the influence of system errors and to check the sensor performance. Self-validation is applied to increase the reliability of the sensor. Therefore, this intelligent sensor is much more flexible and accurate than a traditional sensor.

Capacitive pressure sensors (CPS) have been widely used in engineering, industrial, scientific and medical applications because of their sensitivity and power dissipation. The changes in capacitance occur due to the deformation of the structure. The magnitude of the deformation is proportional to the ambient pressure, but it also depends on the ambient temperature and any initial stresses produced during manufacturing process, which make CPS non-linear and temperature dependent. To correct its non-linear characteristics and compensate temperature in a dynamic environment, the intelligent CPS based on functional link artificial neural networks (FLANN) was developed. The FLANN contains advanced signal processing technique and learning algorithm [46]. 
Colour sensors have found their applications in many fields such as the electricity industry for recognition and assignment of coloured cords and the automatic test of mounted LED arrays or matrices. In the textile industry, they are used to check colouring processes, while in building materials industry, they are suitable to control compounding processes. Colours have an influence on the process control or quality protection as measuring or controlling variables. Conventional colour sensors detect colour property of objects but decisions or control actions are based on human expertise. Intelligent colour sensors have been developed to equip the colour sensors with decisions ability. Benoit [43] proposes an intelligent colour sensor based on fuzzy technique. The definition of functionalities is coded in a special language called Prototype of Language for linguistic Actuators and Sensors (PLICAS). The functionalities coded in PLICAS are sent over a network to be executed by another computing system. This approach allows adding complex functionalities to usual intelligent instruments. For example, it can be useful to implement diagnosis functions into an intelligent sensor.

Fuzzy processing is programmed by PLICAS. Fig.7 illustrates the distribution of intelligence over a network; the intelligent sensor part contains the LAB calculus and the other measurement services and the PLICAS source code of the fuzzy description and fuzzy aggregation. The colour calibration has not been discussed in this paper although it constitutes a critical issue. On the other side, if large number of colours is used, a dynamic auto-calibration should be implemented, otherwise, it will be a very time consuming and laborious operation. Therefore faster approaches have to be developed.

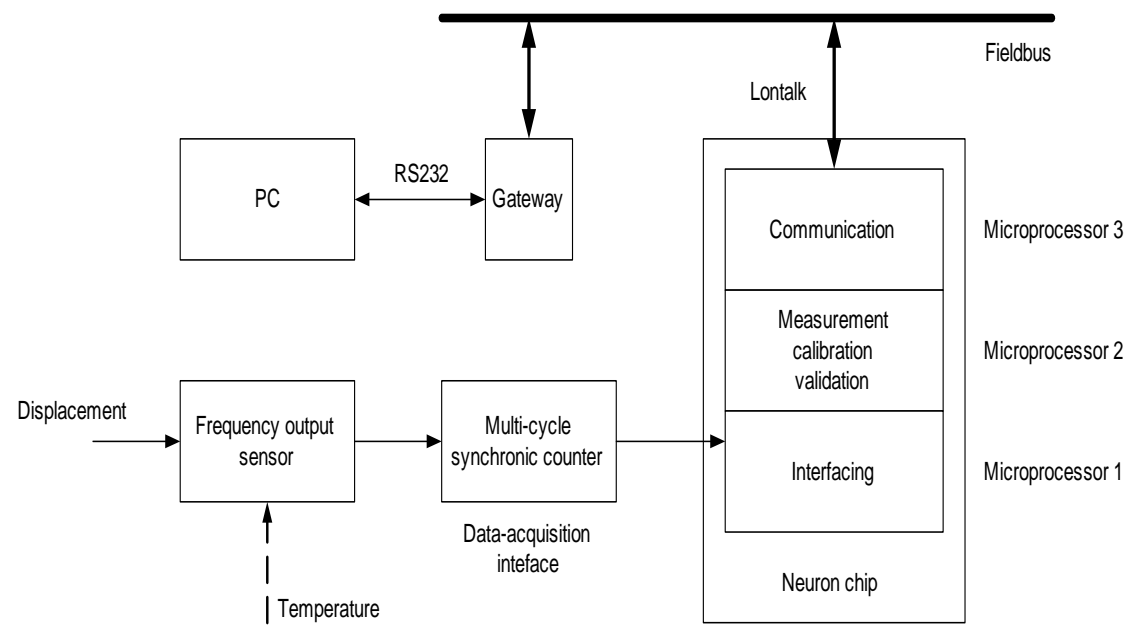

Figure 6. A fieldbus-based intelligent frequency output sensor [18].

Lammerink [47] presents an intelligent gas-mixture flow sensor that can detect the mass and the ratio of two gases in the gas mixture. The sensor mainly comprises different flow sensing elements and an artificial neural network (ANN), as shown in Fig. 8. The sensing elements (SE) are integrated into a chip where the ANN is implemented. The outputs from the sensing elements are transmitted to the ANN to compute the mass and the ratio of two gases in the gas mixture. The sensor output can be communicated with the outside world via RS232 interface. Again, the sensor calibration has not been discussed, although it is an important component. 
The absence of design generalizations and standard interfaces in artificial chemical sensing systems is a well-known problem usually leading to the re-calibration of the olfactory system whenever a sensor has to be substituted.

Bigger problems come up when the migration to sensors based on a different principle is required (e.g. from resistive polymeric sensor to mass-variation quartz crystal microbalance sensor); this necessity involves the re-design of part or even the whole olfactory system forcing research groups to great efforts in system design and software programming. A way to standardize such gas sensors and electronic olfactory systems is presented in [55]. In particular the calibration was proposed to be standardized in IEEE 1451.4. A procedure for dynamic calibration of a sensor gas, for example, was suggested with a calibration transfer function instead of a calibration line [57]. The calibration transfer function enables measurement of the heat generation rates during adsorption, necessary for obtaining a complete picture about adsorption kinetics.

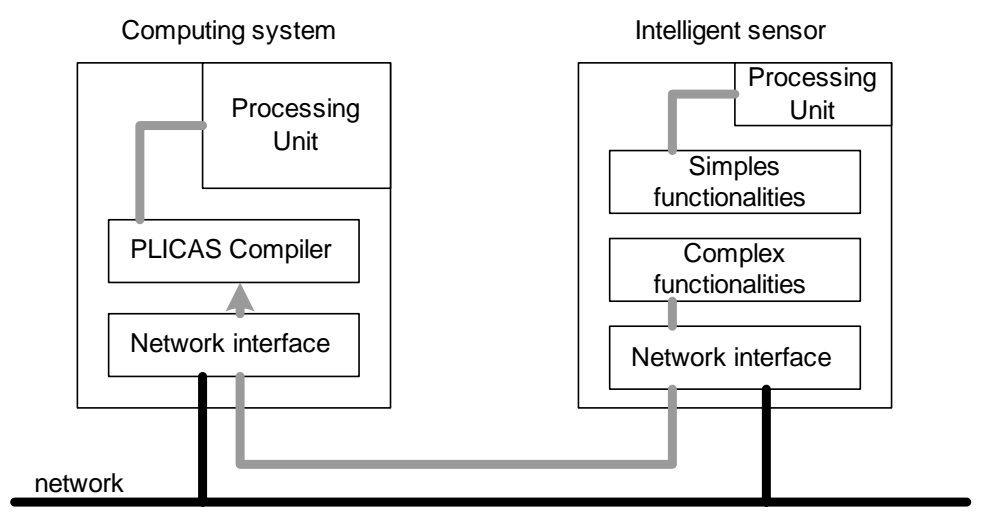

Figure 7. Concept of intelligent fuzzy sensor with small processor [27].

According to the application reviewed above, the development of intelligent sensors has been progressing through improvement of the processing element. Using fuzzy or neural networks promotes sensor flexibility, self-compensation, self-validation, self organization functionalities [42]. Also, integrating all elements into one single chip has contributed to the miniaturization of the sensors. Besides size-reduction and improving processing performance, other elements in the intelligent sensors have also been developed. For example, measurement with in-situ data processing increases accuracy, stability and reliability of the sensing elements. Self-powered technology can enhance power consumption property. Communication technology such as wireless, Bluetooth and infrared (IR) extends communication range.

Uncertainty may be questioned when using fuzzy logic in those smart sensors. The Hybrid Fuzzy Classifier System (HFCS) [56] aims to combine information from multiple domains in order to detect, isolate, identify, and mitigate threats to power networks. The HFCS includes a component that observes the system to learn its normal operating states. Another part of the HFCS contains a database of information including component reliability, sensor accuracy and maintenance records (Fig. 9). 


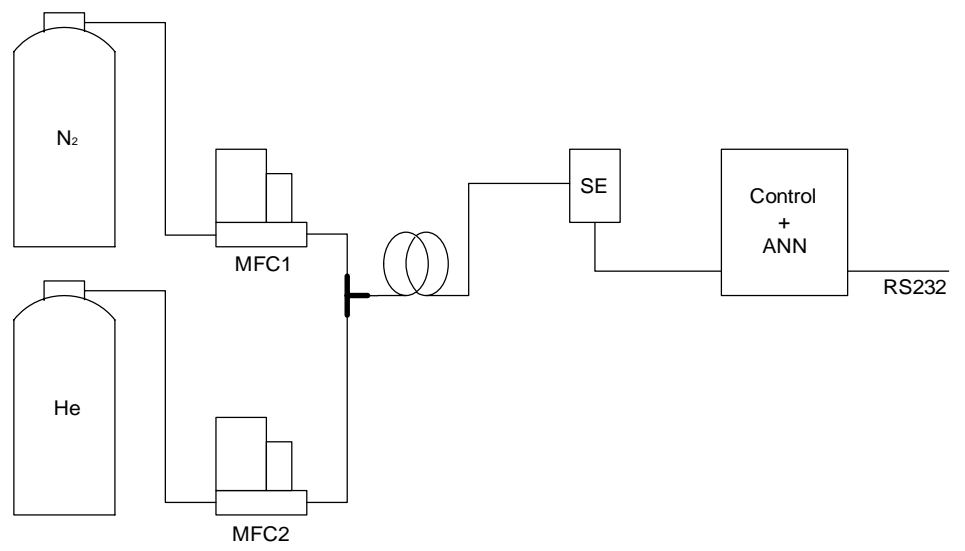

Figure 8. Experimental set-up for the intelligent gas-mixture flow sensor [47].

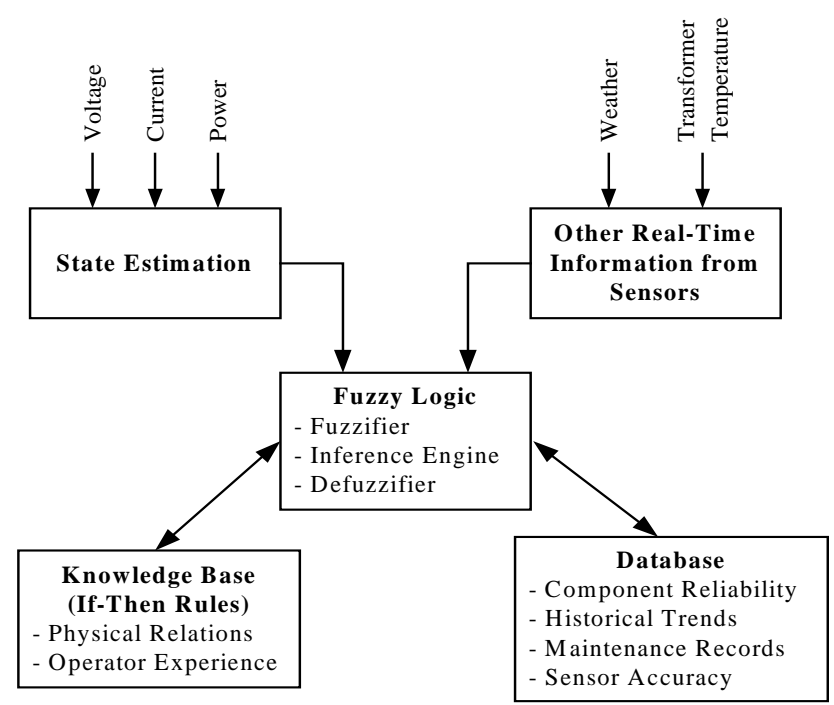

Figure 9. Hybrid Fuzzy Classifier System (HFCS).

For the sensor measurements, four fuzzy inputs (variables) were created: Sensor Reliability, Standard Error, Load Difference and Relative Age. Because the sensor accuracy is explicitly included in the state estimator, it is not included as a fuzzy variable. The hybrid fuzzy classifier system (HFCS) has the potential to increase the robustness of detecting, identifying and characterizing inconsistencies in sensory data from the power system. Some tests have shown very good accuracy in measurements, however, limitations for higher accuracies should be investigated.

In-process measurement sensors [48] have introduced a new aspect of measurement in CNC machines, i.e. 'measuring geometric forms of a workpiece while it is being machined'. This improves on-line error compensation by adjusting machining parameters. A cylindrical capacitance-type spindle displacement sensor was developed [49]. The results from the research showed that the developed sensor could be utilized as an effective and cheap on-line sensing device to monitor cutting conditions and tool performance in the unmanned centre. Novel fractal estimation methodology for online estimation of cutting tool flank wear is presented. The fractal dimensions of the attractor of machining dynamics are extracted from a collection of sensor signals using a suite of signal processing methods comprising wavelet representation and signal separation, and, are related to the instantaneous flank 
wear using a recurrent neural network [50, 51]. Current in-process sensors are based on interferometric and moiré techniques. Vision systems can be integrated with other coordinate digitizing sensors, such as touch probe sensors to acquire at high speed high precision coordinates acquisition of complex features in mechanical components [52, 53].

The MEMS sensors and actuators are widely used in various difficult applications due to their advantages in miniaturisation, low power consumption, ease of measurement and telemetry. Nagel [8] has reviewed sensors made with MEMS. Few examples are cited such as: a smart pen contains three accelerometers, three force sensors and two tilt sensors for signature verification. A smart wristwatch with temperature, pressure, humidity and acceleration sensors is used to monitor an environment. Most of the predictions expected in the last decade have already been implemented (e.g. self calibration, wireless communication) but still time consuming tasks.

The realization of future complete intelligent sensors depends on the development of both hardware and software including electronics and sensing element. Intelligent sensors and actuators will form a network that is able to sense, process, communicate the information, act, and eventually modify system conditions based on this information. The intelligent network will mainly comprise intelligent sensors and actuators, communication channels and an advanced centralized processor. When the intelligent sensor network is becoming available, the ultimate goal to imitate human abilities may become a reality. Figure 12 shows the overall future trend of the use of intelligent sensors architecture in a manufacturing system using sensors clusters deployed in sensitive locations such as hazard warning applications with sensor agent as well as an object agent in closed loop structure.

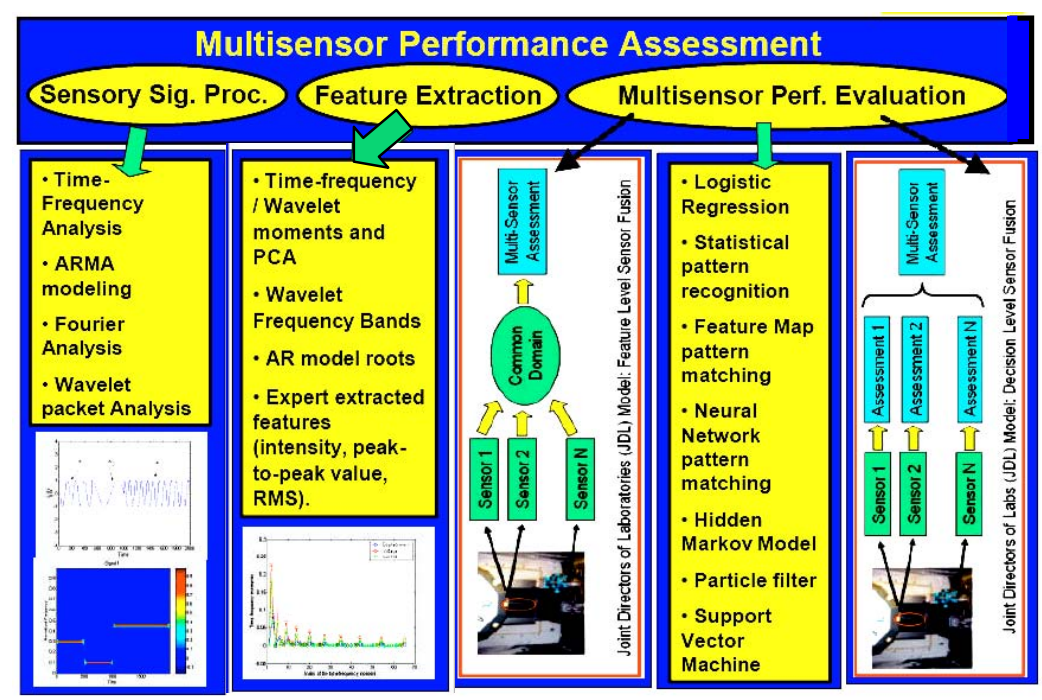

Figure 10. Multi sensor performance assessment.

A particular example of intelligent agent in self maintenance that could be applied to machine tool centres was proposed by Lee [54] and is shown in figure 10. An aspect of the machine performance is assessed by Multi-sensors acquiring signals and failures are predicted after feature recognition is established. It is expected to include such an agent in the next generation of CNC machine-tool centres to adopt the following architecture proposed in figure 11. 


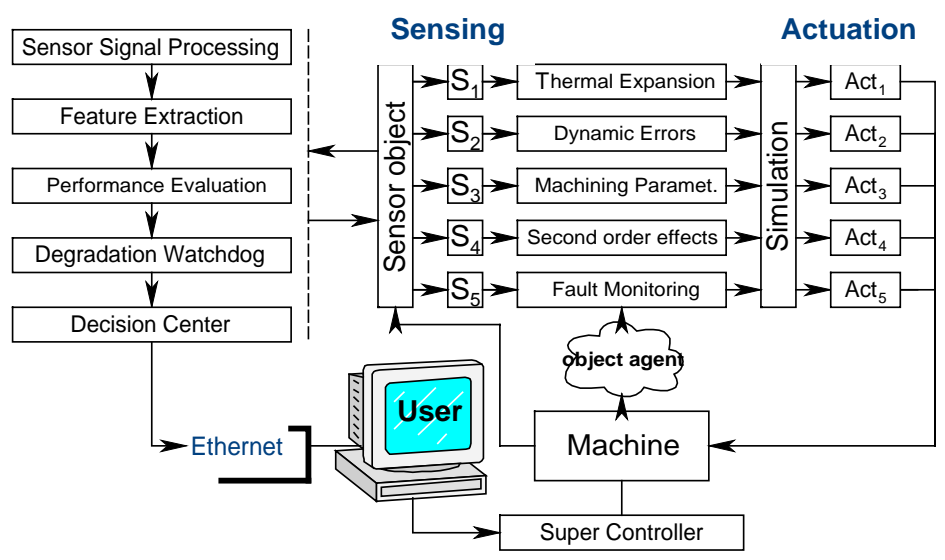

Figure 11. Intelligent sensor cluster activity for the next NC machines generation.

\section{5 - Future requirements for a full process control}

\subsection{Sensor Master/clusters set-up for single/hybrid processes}

For simple and reconfigurable systems, 3D measurements will be possible to govern existing complex phenomena including those parameters probably not required in the equation (fig.12). Selfcalibration will be easier because of the multiplicity of the sensors tracking the same parameter.

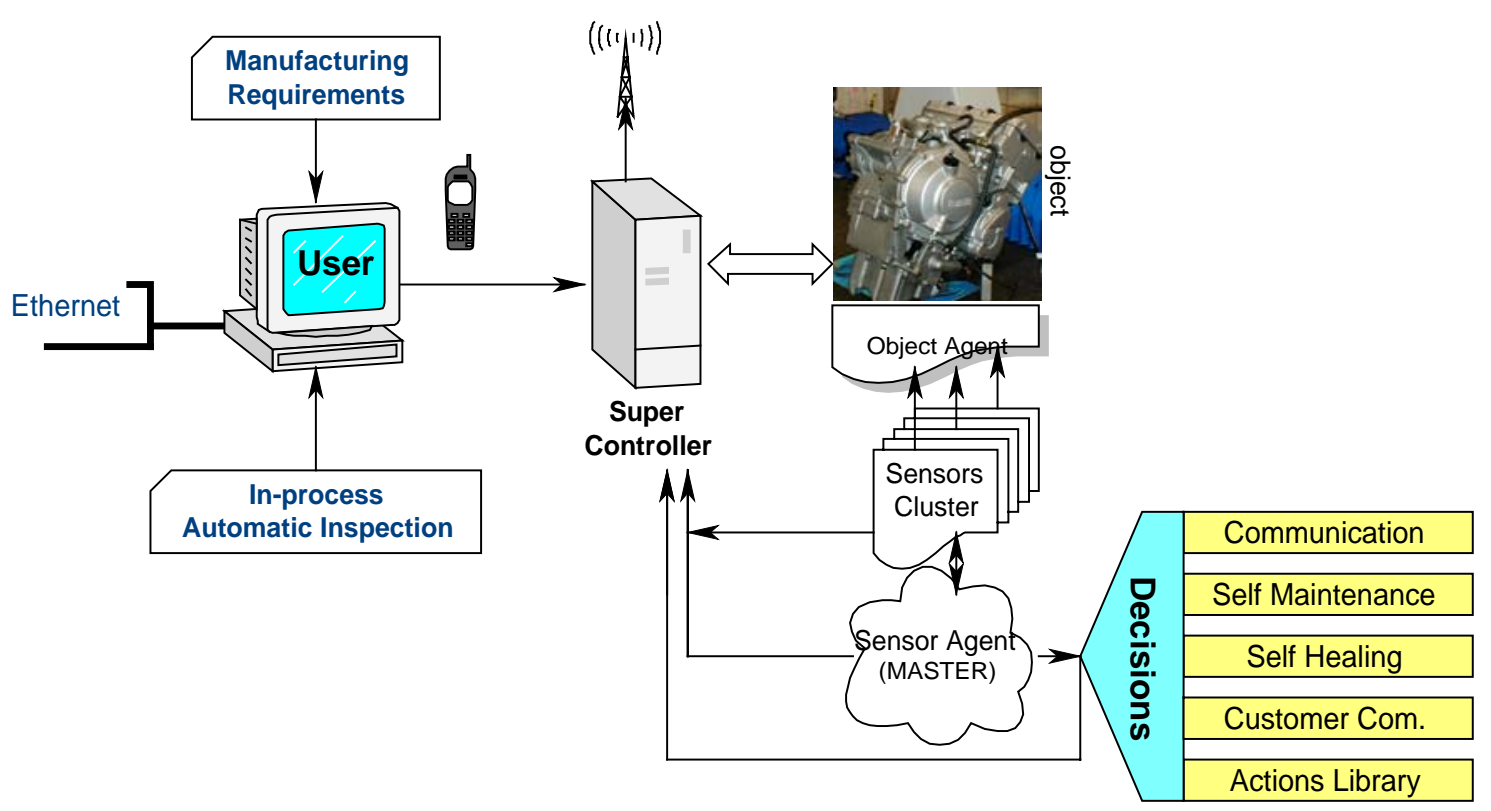

Figure 12. Intelligent sensor cluster activity.

\subsection{In process Parameter evaluation}

In autonomous process systems, the sensing system should be able to track the failing process parameters in real time and apply self adjustment according to requirements. In more optimistic situations, the cluster could capture high order effects that may degrade the performance of the current process. 


\subsection{Extreme environment sensors for manufacturing processes and cutting interfaces}

This is an extremely important issue required in manufacturing at standard level to monitor the cutting interfaces; e.g. laser processing, high speed machining. It becomes extremely important and challenging at nanoscale machining.

\subsection{Plug and Play sensors with standardization of interfaces}

International standardisation of interfaces is very welcomed in industry; this will ease the implementation of plug and play characteristic through expert systems to identify the opposite interface.

\subsection{Scalability and implementation in micro-systems}

Intelligent sensors are not restricted to macro scale applications but extended to micro systems too. The concepts discussed earlier could be downscaled and used in various areas such as biological sensor in micro-fluidics systems and tactile sensors to simulate human fingertip [58].

\section{Conclusion}

An intelligent sensor network is an array of diverse types of sensors in a network tracking a number of variables. With the complexity trend in the new advanced intelligent machines, many characteristics are required beyond the current capabilities of smart sensors.

Fuzzy or neural networks are implemented to ease complex intelligent functionalities, such as selfcompensation, self-validation and computing functionality. With appropriate decision algorithms, the intelligent sensor network should be able to act independently according to information received or adjust itself to the changing working conditions. This will achieve a high reliability closed loop design process. Scalable systems are required to respond urgently to needs with the ever increasing complexity and to survive if part of the network becomes non-reliable or destroyed.

In ultra high precision measurements with very tight accuracies, calibration may constitute a real challenge especially with sensors cluster having large number of measurands to be calibrated, but on the other side, large variety of sensors nodes could be a potential for greater accuracy of information. The reliability could be increased if the resolution is improved without necessarily increase the network traffic.

With such a development of sensor clusters, this will constitute a new key enabling technology for autonomous machines and ability to achieve "just in time" actions required in various manufacturing applications such as zero-defects production and zero downtime. For improved capabilities, the theory of error propagation including higher order uncertainties is expected to be used to track down nonlinearities and sources of errors present in any systems. This method will be enabled with intelligent cluster sensors. Robustness and high accuracy of information are of paramount importance in such systems. Needless to mention that sensor location although out of the scope within this paper, is extremely important. 


\section{Acknowledgments}

The author acknowledges gratefully the support of the European Network of Excellence on Intelligent Production Machines and Systems (I*PROMS) for their support of this work. The author wishes to extend his thanks to the referees for their suggestions.

\section{References and Notes}

1. Burl, M.C., Review of Literature on Smart Sensors, 1998, in http://wwwaig.jpl.nasa.gov/public/mls/home/burl/smart_sensors.html (accessed 03/2005).

2. Najafi, K., Smart Sensors, J. Micromech. \& Microeng., 1991,1, pp. 86-102,

3. Expanding the Vision of Sensor Materials, National Materials Advisory Board, National Academic Press, Washington, D.C., 1995.

4. Schodel H., Utilization of fuzzy techniques in intelligent sensors, Fuzzy Sets and Systems, 1994, vol. 63, pp. 271-292.

5. Brignell J. E., The future of intelligent sensors: a problem of technology or ethics?, Sensors and Actuators A, 1996, vol. 56, pp. 11-15,.

6. Chita M.-A., Anghel S. and Iorga-Siman I., Some aspects concerning optically powered intelligent sensors, Proceedings of SPIE, 2000, vol. 4068, pp. 633-638.

7. Takahashi K. and Nozaki S., From intelligent sensors to fuzzy sensors, Sensors and Actuators A, 1994, vol. 40, pp. 89-91.

8. Nagel D. J., Microsensor clusters, Microelectronics Journal, 2002, vol. 33, pp. 107-119.

9. Glynme-Jones P., Tudor M. J., Beeby S. P., and White N. M., An electromagnetic, vibrationpowered generator for intelligent sensor systems, Sensors and Actuators A, 2004, vol. 110, pp. 344-349.

10. James E. P., Tudor M. J., Beeby, S. P., Harris, N. R., Glynne-Jones, P., Ross, J. N., and White, N. M., An investigation of self-powered systems for condition monitoring applications, Sensors and Actuators A, 2004, vol. 110, pp. 171-176.

11. Mitcheson, P. D., P. Miao, B. H. Stark, E. M. Yeatman, Holmes, A. S., and Green, T. C., MEMS electrostatic micropower generator for low frequency operation, Sensors and Actuators A, 2004, vol. 115, pp. 523-529.

12. Roundy, S., Wright, P. K., and Rabaey, J., A study of low level vibrations as a power source for wireless sensor nodes, Computer Communications, 2003, vol. 26, pp. 1131-1144.

13. Al-Mohanadi M. R., Ross, J. N., and Brignell, J. E., Optical power and intelligent sensors, Sensors and Actuators A, 1997, vol. 60, pp. 142-146.

14. Jr, B. F. S., Ruiz-Sandoval, M. E., and Kurata, N., Smart sensing technology: opportunities and challenges, Structural Control and Health Monitoring, 2004, vol. 11, pp. 349-368.

15. Vaillant, O. R. and Garcia, C., PC-based natural gas flow computer using intelligent instrumentation and field bus, Measurement, 2003, vol. 33, pp. 259-271.

16. Robert, M., Riviere, J. M., Noizette, J. L., and Hermann, F., Smart sensors in flexible manufacturing systems, Sensors and Actuators A, 1993, vol. 37-38, pp. 239-246.

17. Meijer, G. C. M., Concepts and focus point for intelligent sensor systems, Sensors and Actuators A, 1994, vol. 41-42, pp. 183-191. 
18. Tian, G. Y., Zhao, Z. X., and Baines, R. W., A fieldbus-based intelligent sensor, Mechatronics, 2000, vol. 10, pp. 835-849.

19. Liu, J.-G., Self-correction algorithms and applications to digital signal processing, Measurement, 2002, vol. 31, pp. 107-116.

20. Sorrentino, R., OLC - a signal conditioning and calibration technique for Magnetoelastic Sensors, Proceedings of SPIE, 2004, vol. 5384, pp. 108-119.

21. Gao, Y. and Radha, H., A multistage camera self-calibration algorithm, Proceedings- IEEE International Conference on Acoustics, Speech, and Signal Processing, 2004, vol. 3, pp. 537-540.

22. Taner, A. H. and Brignell, J. E., Aspects of intelligent sensor reconfiguration, Sensors and Actuators A, 1995, vol. 46-47, pp. 525-529.

23. Kiyono, S., Arai, Y. and Chetwynd, D., Self-calibration of sensors in software datum methods, $4^{\text {th }}$ Int. EUSPEN conf. Glasgow, 2004.

24. Clarke, D. W., Intelligent instrumentation, Transactions of the Institute of Measurement and Control, 2000, vol. 22, pp. 3-27.

25. Nelson, C. L. and Fitzgerald, D. S., Sensor fusion for intelligent alarm analysis, IEEE Annual International Carnahan Conference on Security Technology, 1996, pp. 143-150.

26. Johnson, R. N. and Woods, S. P., Overview and Status Update for the IEEE 1451.2: Transducer to Microprocessor Communications Protocols and Transducer Electronics Data Sheet (TEDS) Formats, Proceeding Sensor Expo 2000, Anaheim, CA, p. 17-24, May 9, 2000.

27. IEEE Std 1451.2-1997, IEEE Standard for a Smart Transducer Interface for Sensors and Actuators - Transducer to Microprocessor Communication Protocols and Transducer Electronic Data Sheet (TEDS) Formats, IEEE Instrumentation and measurement Society, TC-9 Committee on Sensor Technology, Institute of Electrical and Electronics Engineers, New York, N.Y., Sept. 1998.

28. Wiczer, J., Smart Interfaces for Sensors. Proceeding Sensor Expo 2001, 2001 Chicago, IL, June.

29. Bradshaw, J. M., Software Agents, 1997, AAAI Press / MIT Press.

30. Jennings, N. J. and Wooldridge, M.J. (1998), Applications of Intelligent Agents, In Agent Technology - Foundations, Applications, and Markets, Jennings, N. R. and Wooldridge, M. J., Ed., Springer-Verlag, Berlin, Germany, 1977, pp. 3-28.

31. Nwana, H.S., Software Agents: An Overview, Knowledge Engineering Review, 1996, 11, 3, 1-40.

32. Andersson L.and Rönnbom Å., Intelligent Agents- A New Technology for Future Distributed Sensor Systems?, MSc thesis, Göteborg University, 1999

33. Petrie, C. J., “Agent-Based Engineering, the Web, and Intelligence”, IEEE Expert/Intelligent Systems \& Their Applications , 1996, 11, 6, pp. 24-29.

34. Brustoloni, Jose C. , "Autonomous Agents: Characterization and Requirements," Carnegie Mellon Technical Report CMU-CS-91-204, 1991, Pittsburgh: Carnegie Mellon University

35. Franklin, Stan (), Artificial Minds, Cambridge, MA: MIT Press. ,1995

36. M.Coen, http://www.ai.mit.edu/people/sodabot/slideshow/total/P001.html

37. Maes, Pattie, "Artificial Life Meets Entertainment: Life like Autonomous Agents," 1995, Communications of the ACM, 38, 11, 108-114

38. Nwana, H.S., Software Agents: An Overview, Knowledge Engineering Review, 1996, 11, 3, 1-40. 
39. Foner, L.N. "What's an agent anyway?”, Proceedings of the First International Conference on Autonomous Agents (AA`97), 1997.

40. Hayes-Roth, B., “An Architecture for Adaptive Intelligent Systems”, ArtificialIntelligence: special issue on agents and interactivity, 1995, 72, pp.329-365.

41. Stone, P. and Veloso, M., "Multi-agent Systems: A survey from a machine learning perspective”, 1997, Technical Report 193, Department of Computer Science, Carnegie Mellon University, Pittsburgh, PA.

42. Collier, T. C. and Taylor C., Self-organization in sensor networks, Journal of Parallel and Distributed Computing, 2004, vol. 64, pp. 866-873.

43. Benoit, E. and Foulloy, L., High functionalities for intelligent sensors, application to fuzzy colour sensor, Measurement, 2001, vol. 30, pp. 161-170.

44. M.Bourahla and M.Benmohamed Formal Specification and Verification of Multi-Agent Systems, Electronic Notes in Theoretical Computer Science, Volume 123, 1 March 2005, pp. 5-17.

45. Y.-E. Nahm and H. Ishikawa A hybrid multi-agent system architecture for enterprise integration using computer networks, Robotics and Computer-Integrated Manufacturing, Volume 21, Issue 3, June 2005, pp.217-234.

46. Patra, J. C. and Bos A. V. D., Modeling of an intelligent pressure sensor using functional link artificial neyral networks, 2000, ISA Transactions, vol. 39, pp. 15-27.

47. Lammerink, T. S. J., Dijkstra, F., Houkes, Z., and Kuijk J. V., Intelligent gas-mixture flow sensor, 1995, Sensors and Actuators A, vol. 46-47, pp. 380-384.

48. Vacharanukul, K., Mekid, S., In-process dimensional measurement sensors, 2005, Measurement, volume 38, pp. 204-218.

49. Kim I.H., Jang D.Y., Kim W.J., Han D.C., In-process sensing of tool wear and process states using a cylindrical displacement sensor in hard turning, 2001, Proceedings of the Institution of Mechanical Engineers Part B - Journal of Engineering Manufacture, Vol. 215, 12, 1673-1682

50. Bukkapatnam S.T.S., Kumara S.R.T., Lakhtakia A., Fractal estimation of flank wear in turning, 2000, Journal of Dynamic Systems Measurement and Control - Transactions of the ASME, Vol. 122, 1, pp.89-94.

51. Wang L.T., Mehrabi M.G., KannateyAsibu E., Hidden Markov model-based tool wear monitoring in turning, 2002, Journal of Manufacturing Science and Engineering - Transactions of the ASME, Vol. 124, 3, pp.651-658.

52. Shen T.S., Huang J., Menq C.H., Multiple-sensor Integration for Rapid and High-precision Coordinate Metrology, 2000, IEEE/ASME Transactions on Mechatronics, Vol. 5, 2, 110-121

53. Nashman M., Vision and touch Sensors for Dimensional Inspection, 1993, Manufacturing Review, Vol.6, 2, 155-162.

54. Lee, J., Smart prognostic and predictive maintenance systems course, 2004, Center for Intelligent Maintenance systems.

55. Ulivieri N., Distante C., Luca T., Rocchi S., Siciliano P., 2006, IEEE1451.4: A way to standardize gas sensor, Sensors and Actuators B, volume 114, pp.141-151.

56. Holbert K.E., Lin K., Reducing State Estimation Uncertainty Through Fuzzy Logic Evaluation of Power System Measurements, 2004, 8th International Conference on Probability Methods Applied to Power Systems, Ames, Iowa, 2004, pp. 205-211. 
57. Petkovska M., Antov D., Zimmermann W., Schein E., Dynamic calibration of a sensor gas calorimeter for measurement of heat generation rates during kinetic of adsorption experiments, 2004, Thermochimica acta, volume 415. pp.21-25.

58. Hauptmann, P. R., Selected examples of intelligent (micro) sensor systems: state-of-the-art and tendencies, Meas. Sci. Technol. 17 No 3 (March 2006) pp.459-466.

(C) 2006 by MDPI (http://www.mdpi.org). Reproduction is permitted for noncommercial purposes. 